\title{
COMPACT EINSTEIN-WEYL FOUR-MANIFOLDS WITH COMPATIBLE ALMOST COMPLEX STRUCTURES
}

\author{
HIROYUKI KAMADA
}

\section{Introduction}

A Weyl manifold is a smooth conformal manifold $(M, C)$ equipped with a torsion-free affine connection $D$ preserving the conformal structure $C$. A Weyl manifold $(M, C, D)$ is said to be Einstein-Weyl if its symmetrized Ricci tensor $r^{D \text { (sym) }}$ is proportional to a metric representative of $C$. The Levi-Civita connection $\nabla$ of an Einstein manifold $(M, g)$ gives an Einstein-Weyl structure $([g], \nabla)$ on $M$, where $[g]$ denotes the conformal structure determined by $g$. Thus the notion of Einstein-Weyl structures is a generalization of Einstein metrics, so there are many studies in this topic (see Pedersen-Swann [9], [10], Itoh [4], and their references).

An almost complex structure $J$ on a conformal manifold $(M, C)$ is said to be compatible if $J$ preserves $C$. Let $(M, C, J)$ be a conformal manifold with a compatible almost complex structure $J$. By making use of the Lee form $\beta_{g}$ of each metric $g$ in $C$, we can naturally define a unique Weyl connection $D$ on $(M, C, J)$, which is called the canonical Weyl connection associated with $(C, J)$. In the 4-dimensional case, we shall call such a quadruple $(M, C, D, J)$ an almost Hermitian-Weyl 4-manifold. It is known that for an almost Hermitian-Weyl 4manifold $(M, C, D, J), J$ is integrable if and only if $J$ is parallel with respect to $D$. When $J$ is $D$-parallel, $(M, C, D, J)$ is called a Hermitian-Weyl manifold. Note that the definition of (almost) Hermitian-Weyl manifolds is very similar to that of (almost) Kähler manifolds. An almost Hermitian-Einstein-Weyl 4-manifold means an almost Hermitian-Weyl 4-manifold whose Weyl structure is EinsteinWeyl.

Sekigawa [6] showed that any compact almost Kähler-Einstein manifold with nonnegative scalar curvature must be Kähler-Einstein. Motivated by his result, we shall consider the integrability problem for almost Hermitian-Einstein-Weyl 4-manifolds. Our main result is as follows:

The author was supported by Grant-1n-Aid for Encouragement of Young Scientists (No. 09740075), The Ministry of Education, Science, Sports and Culture, Japan.

Received October 9, 1998; revised February 3, 1999. 
THEOREM 1.1. A compact almost Hermitian-Einstein-Weyl 4-manifold with nonnegative conformal scalar curvature must be Hermitian-Einstein-Weyl.

\section{Almost Hermitian-Einstein-Weyl structures}

Let $(M, C, D)$ be a 4-dimensional Weyl manifold. Then for any metric $g$ in $C$, there exists a 1 -form $\omega_{g}$ such that $D g=\omega_{g} \otimes g$. We note that $d \omega_{g}$ is independent of the choice of $g \in C$. Indeed, for another metric $g^{\prime}=e^{f} g$ in $C$, the corresponding 1 -forms $\omega_{g}$ and $\omega_{g^{\prime}}$ satisfy the following:

$$
\omega_{g^{\prime}}=\omega_{g}+d f, \quad d \omega_{g}=d \omega_{g^{\prime}}(=: d \omega) .
$$

Denote respectively by $R^{D}, r^{D}$ and $s_{g}^{D}$ the curvature tensor, the Ricci curvature and the conformal scalar curvature of $D$ with respect to $g$ in $C$ :

$$
\begin{gathered}
R^{D}(X, Y) Z:=D_{X}\left(D_{Y} Z\right)-D_{Y}\left(D_{X} Z\right)-D_{[X, Y]} Z, \\
r^{D}(X, Y):=\operatorname{tr}\left(V \mapsto R^{D}(V, Y) X\right), \quad s_{g}^{D}:=\operatorname{tr}_{g}\left(r^{D}\right), \quad s^{D}:=s_{g}^{D} g .
\end{gathered}
$$

Note that the Ricci tensor $r^{D}$ is not necessarily symmetric. We then denote by $r^{D \text { (sym) }}$ and $r^{D \text { (skew) }}$ the symmetric and skewsymmetric parts of $r^{D}$, respectively:

$$
\begin{aligned}
& r^{D(\text { sym })}(X, Y):=\frac{1}{2}\left(r^{D}(X, Y)+r^{D}(Y, X)\right), \\
& r^{D \text { (skew) }}(X, Y):=\frac{1}{2}\left(r^{D}(X, Y)-r^{D}(Y, X)\right) .
\end{aligned}
$$

It is known that the skewsymmetric part $r^{D(\text { skew })}$ is given by $r^{D(\text { skew })}=-d \omega$.

The curvature tensor $R^{D}$ decomposes as

$$
R^{D}=W_{+} \oplus W_{-} \oplus r_{0}^{D(\text { sym })} \oplus r_{+}^{D(\text { skew })} \oplus r_{-}^{D(\text { skew })} \oplus s^{D},
$$

where $W_{ \pm}$are the self-dual and anti-self-dual parts of the Weyl conformal curvature tensor, $r_{0}^{D(\mathrm{sym})}$ is the traceless part of $r^{D(\mathrm{sym})}$, and $r_{ \pm}^{D(\mathrm{skew})}$ are the selfdual and anti-self-dual parts of $r^{D \text { (skew) }}$ (see Pedersen-Swann [9]).

A Weyl manifold $(M, C, D)$ is said to be Einstein-Weyl if the symmetric part $r^{D \text { (sym) }}$ of the Ricci tensor is proportional to a metric $g$ in $C$ :

$$
r^{D(\operatorname{sym})}=\frac{s_{g}^{D}}{4} g .
$$

Unlike the Einstein case, the conformal scalar curvature $s_{g}^{D}$ is not constant in general; however, the sign of $s_{g}^{D}$ is well-defined for compact Einstein-Weyl 4manifolds (cf. Pedersen-Swann [10], Itoh [4]).

We next consider almost complex structures on Weyl manifolds. Let $(M, C, D)$ be a 4-dimensional Weyl manifold and $J$ an almost complex structure on $M$. Suppose that $J$ preserves $C$, i.e., $g(J X, J Y)=g(X, Y)$ for any metric $g$ in 
$C$. The fundamental form $\Omega_{g}$ of $(g, J)$ is now defined by $\Omega_{g}(X, Y):=g(J X, Y)$. It follows from the peculiarity of the 4-dimensional case that there exists a 1-form $\beta_{g}$, called the Lee form of $(M, g, J)$, satisfying

$$
d \Omega_{g}=\beta_{g} \wedge \Omega_{g}
$$

In particular, the exterior derivative $d \beta_{g}$ of the Lee form is orthogonal to the fundamental form $\Omega_{g}$ :

$$
d \beta_{g} \wedge \Omega_{g} \equiv 0 .
$$

For another metric $g^{\prime}=e^{f} g$ in $C$, the Lee forms $\beta_{g}$ and $\beta_{g^{\prime}}$ satisfy the following:

$$
\beta_{g^{\prime}}=\beta_{g}+d f, \quad d \beta_{g^{\prime}}=d \beta_{g} .
$$

Comparing (2.1) with (2.5), we see that $\beta_{g}-\omega_{g}$ is independent of the choice of $g$.

If $D$ is the canonical Weyl connection, i.e., $\beta_{g} \equiv \omega_{g}$, then $(M, C, D, J)$ is called an almost Hermitian-Weyl manifold. Furthermore, $(M, C, D, J)$ is said to be almost Hermitian-Einstein-Weyl if $(C, D)$ is also Einstein-Weyl. An almost Hermitian-Weyl manifold $(M, C, D, J)$ is said to be Hermitian-Weyl if $D J \equiv 0$.

Proposition 2.1. $(M, C, D, J)$ is an almost Hermitian-Weyl 4-manifold if and only if $(g, D, J)$ satisfies

$$
g\left(\left(D_{X} J\right) Y, Z\right)+g\left(\left(D_{Y} J\right) Z, X\right)+g\left(\left(D_{Z} J\right) X, Y\right) \equiv 0,
$$

where $g$ is a metric in $C$. Furthermore, if $(M, C, D, J)$ is an almost HermitianWeyl manifold, then the following holds

$$
\left(D_{J X} J\right) J Y+\left(D_{X} J\right) Y \equiv 0 .
$$

In particular, the g-trace $\operatorname{tr}_{g}(D J)$ of $(X, Y) \mapsto\left(D_{X} J\right) Y$ is identically zero:

$$
\operatorname{tr}_{g}(D J) \equiv 0
$$

Proof. By definition, the covariant derivative $D \Omega_{g}$ of the fundamental form $\Omega_{g}$ satisfies

$$
\left(D_{X} \Omega_{g}\right)(Y, Z)=g\left(\left(D_{X} J\right) Y, Z\right)+\omega_{g}(X) g(J Y, Z) .
$$

Since $D$ is torsion-free, we have

$$
\begin{aligned}
d \Omega_{g}(X, Y, Z) & =\Im_{X, Y, Z}\left(D_{X} \Omega_{g}\right)(Y, Z) \\
& =\Im_{X, Y, Z}\left\{g\left(\left(D_{X} J\right) Y, Z\right)+\omega_{g}(X) \Omega_{g}(Y, Z)\right\} \\
& =\left(\omega_{g} \wedge \Omega_{g}\right)(X, Y, Z)+\Im_{X, Y, Z} g\left(\left(D_{X} J\right) Y, Z\right),
\end{aligned}
$$

where $\Im_{X, Y, Z}$ denotes the cyclic summation with respect to $X, Y, Z$. It then follows that $(M, C, D, J)$ is an almost Hermitian-Weyl manifold if and only if $(g, D, J)$ satisfies (2.6). 
In order to show (2.7), we note that

$$
\left(D_{X} J\right) J Y=-J\left(D_{X} J\right) Y, \quad g\left(\left(D_{X} J\right) Y, Z\right)=-g\left(Y,\left(D_{X} J\right) Z\right) .
$$

By using (2.6) and (2.9), we have

$$
\begin{array}{r}
g\left(\left(D_{X} J\right) Y, Z\right)+g\left(\left(D_{Y} J\right) Z, X\right)+g\left(\left(D_{Z} J\right) X, Y\right) \equiv 0 \\
g\left(\left(D_{X} J\right) Y, Z\right)-g\left(\left(D_{J Y} J\right) J Z, X\right)-g\left(\left(D_{J Z} J\right) X, J Y\right) \equiv 0 \\
g\left(\left(D_{J X} J\right) Y, J Z\right)-g\left(\left(D_{Y} J\right) Z, X\right)+g\left(\left(D_{J Z} J\right) J X, Y\right) \equiv 0 \\
g\left(\left(D_{J X} J\right) J Y, Z\right)+g\left(\left(D_{J Y} J\right) Z, J X\right)-g\left(\left(D_{Z} J\right) X, Y\right) \equiv 0 .
\end{array}
$$

Taking summation of these, we have

$$
2 g\left(\left(D_{X} J\right) Y+\left(D_{J X} J\right) J Y, Z\right) \equiv 0 .
$$

This shows (2.7). By taking $g$-trace of (2.7), we immediately obtain (2.8).

From Proposition 2.1, we may regard an almost Hermitian-Weyl manifold as a conformal geometric analogue to almost Kähler one. Indeed, our results for almost Hermitian-Weyl 4-manifolds can be proved by making use of arguments similar to those in almost Kähler geometry (cf. Sekigawa [6], Draghici [1]).

As in almost Hermitian geometry, we introduce the notion of the $*$-Ricci tensor $r^{D *}$ and the $*$-scalar curvature $s^{D *}$ of $(C, D, J)$ :

$$
r^{D *}(X, Y):=\operatorname{tr}\left(V \mapsto R^{D}(Y, J V) J X\right), \quad s_{g}^{D *}:=\operatorname{tr}_{g}\left(r^{D *}\right),
$$

where $g$ is a metric representative of $C$.

For a $(0,2)$-tensor field $t$ on $(M, C, D, J)$, we denote respectively by $t^{\text {(sym) }}$ and $t^{\text {(skew) }}$ the symmetric and skewsymmetric parts of $t$, and also denote by $t^{\text {(inv) }}$ and $t^{(\text {anti) }}$ the $J$-invariant and $J$-anti-invariant parts of $t$ :

$$
\begin{aligned}
t^{(\mathrm{inv})}(X, Y) & :=\frac{1}{2}(t(X, Y)+t(J X, J Y)), \\
t^{(\text {anti) }}(X, Y) & :=\frac{1}{2}(t(X, Y)-t(J X, J Y)) .
\end{aligned}
$$

On the space of 2-forms, we obtain the following orthogonal decomposition:

$$
\bigwedge^{2} T^{*} M=\bigwedge_{+} \oplus \bigwedge_{-} ; \quad \bigwedge_{+}=R \Omega_{g} \oplus \bigwedge^{(\mathrm{anti})}, \quad \bigwedge_{-}=\bigwedge_{0}^{(\mathrm{inv})}
$$

where $\bigwedge_{ \pm}, \boldsymbol{R} \Omega_{g}, \bigwedge_{0}^{(\text {inv })}$ and $\bigwedge^{(\text {anti) }}$ denote respectively self-dual and anti-self-dual 2-forms, multiples of the fundamental form $\Omega_{g}$, the traceless $J$-invariant 2-forms and the $J$-anti-invariant 2 -forms.

For simplicity, we set 


$$
\begin{aligned}
t^{\text {(sym.nnv) }}(X, Y) & :=\frac{1}{4}(t(X, Y)+t(Y, X)+t(J X, J Y)+t(J Y, J X)), \\
t^{\text {(sym.anti) }}(X, Y) & :=\frac{1}{4}(t(X, Y)+t(Y, X)-t(J X, J Y)-t(J Y, J X)), \\
t^{\text {(skew.nnv) }}(X, Y) & :=\frac{1}{4}(t(X, Y)-t(Y, X)+t(J X, J Y)-t(J Y, J X)), \\
t^{\text {(skew.anti) }}(X, Y) & :=\frac{1}{4}(t(X, Y)-t(Y, X)-t(J X, J Y)+t(J Y, J X)) .
\end{aligned}
$$

If we define a tensor field $\tau$ associated with a given $(0,2)$-tensor field $t$ by $\tau(X, Y):=t(J X, Y)$, then the following hold:

$$
\begin{gathered}
\tau^{\text {(sym.nnv) }}(X, Y)=t^{\text {(skew.nnv) }}(J X, Y), \tau^{\text {(sym.anti) }}(X, Y)=t^{\text {(sym.anti) }}(J X, Y), \\
\tau^{\text {(skew.nnv) }}(X, Y)=t^{\text {(sym.nnv) }}(J X, Y), \tau^{\text {(skew.anti) }}(X, Y)=t^{\text {(skew.anti) }}(J X, Y) .
\end{gathered}
$$

The $J$-invariant parts $r^{D *(\text { sym.nv) }}$ and $r^{D \text { (sym.nnv) }}$ of the symmetrized $*$-Ricci and Ricci tensors of $D$ satisfy the following relation:

Proposition 2.2. For an almost Hermitian-Weyl 4-manifold $(M, C, D, J)$, we have the following formulae:

$$
\begin{aligned}
r^{D *(\text { sym.ınv) }}(X, Y) & =r^{D \text { (sym.ınv) }}(X, Y)+\frac{1}{2} B(X, Y) \\
s_{g}^{D *} & =s_{g}^{D}+\frac{1}{2}|D J|_{g}^{2},
\end{aligned}
$$

where $B$ is defined by $B(X, Y):=\operatorname{tr}_{g} g((D J) X,(D J) Y)$.

Proof. We first recall the definition of the second covariant derivative of $J$ :

$$
\left(D_{X} D_{Y} J\right) Z:=D_{X}\left(\left(D_{Y} J\right) Z\right)-\left(D_{D_{X}} J\right) Z-\left(D_{Y} J\right) D_{X} Z \text {. }
$$

By definition, we have the following formulae:

LEMMA 2.3. Let $(M, C, D)$ be a Weyl manifold with a compatible almost complex structure $J$ and $g$ a metric representative of $C$. Then $(g, D, J)$ satisfies

$$
\begin{gathered}
g\left(\left(D_{X} D_{Y} J\right) U, V\right)+g\left(U,\left(D_{X} D_{Y} J\right) V\right) \equiv 0, \\
\left(D_{X} D_{Y} J\right) J V+J\left(D_{X} D_{Y} J\right) V=-\left(D_{X} J\right)\left(D_{Y} J\right) V-\left(D_{Y} J\right)\left(D_{X} J\right) V .
\end{gathered}
$$

Furthermore, if $(M, C, D, J)$ is almost Hermitian-Weyl, then we have

$$
g\left(\left(D_{V} D_{X} J\right) Y-\left(D_{V} D_{Y} J\right) X, U\right)=-g\left(\left(D_{V} D_{U} J\right) X, Y\right) \text {. }
$$

We next recall the following curvature identity, so-called the Ricci identity:

$$
R^{D}(X, Y) J V-J R^{D}(X, Y) V=\left(D_{X} D_{Y} J\right) V-\left(D_{Y} D_{X} J\right) V .
$$


By the definitions of $r^{D}$ and $r^{D *}$ and the Ricci identity (2.15), we have the following:

$$
r^{D *}(X, Y)=r^{D}(X, Y)-\sum_{A} g\left(\left(D_{A} D_{Y} J-D_{Y} D_{A} J\right) e_{A}, J X\right)
$$

where $\left\{e_{A}\right\}$ is a $g$-orthonormal frame field and where $D_{A}$ denotes the covariant derivative by $e_{A}$.

Notice that the $g$-trace of a $(1,2)$-tensor field $T$ and the covariant derivative $D T$ of $T$ satisfy

$$
D \operatorname{tr}_{g} T=\operatorname{tr}_{g} D T+\omega_{g} \otimes \operatorname{tr}_{g} T .
$$

By (2.8), we thus obtain

$$
\sum_{A} g\left(\left(D_{Y} D_{A} J\right) e_{A}, J X\right) \equiv 0 .
$$

Applying (2.14) to the term $\sum_{A} g\left(\left(D_{A} D_{Y} J\right) e_{A}, J X\right)$, we see that

$$
r^{D *}(X, Y)=r^{D}(X, Y)+\sum_{A} g\left(\left(D_{A} D_{A} J\right) J X-\left(D_{A} D_{J X} J\right) e_{A}, Y\right) .
$$

On the other hand, it follows from (2.16) and (2.17) that

$$
r^{D *}(J Y, J X)=r^{D}(J Y, J X)+\sum_{A} g\left(\left(D_{A} D_{J X} J\right) e_{A}, Y\right)
$$

From (2.18) and (2.19), we obtain the following:

$$
r^{D *}(X, Y)+r^{D *}(J Y, J X)=r^{D}(X, Y)+r^{D}(J Y, J X)+\sum_{A} g\left(\left(D_{A} D_{A} J\right) J X, Y\right) .
$$

By using (2.13), we obtain (2.10) as follows:

$$
\begin{aligned}
4\left(r^{D *(\text { sym.nnv })}(X, Y)-r^{D(\text { sym.nnv })}(X, Y)\right) \\
=\sum_{A}\left\{g\left(\left(D_{A} D_{A} J\right) J X, Y\right)+g\left(\left(D_{A} D_{A} J\right) J Y, X\right)\right\} \\
=\sum_{A} g\left(J\left(D_{A} D_{A} J\right) Y+\left(D_{A} D_{A} J\right) J Y, X\right) \\
=-2 \sum_{A} g\left(\left(D_{A} J\right)\left(D_{A} J\right) Y, X\right) \\
=2 \sum_{A} g\left(\left(D_{A} J\right) X,\left(D_{A} J\right) Y\right) \\
=2 \operatorname{tr}_{g} g((D J) X,(D J) Y)=2 B(X, Y) .
\end{aligned}
$$

Taking $g$-trace of (2.10), we immediately obtain (2.11).

In the rest of this section, we always assume that $(M, C, D, J)$ is a compact almost Hermitian-Weyl 4-manifold. 
We now consider the first Chern class $c_{1}(M)$ of such a manifold $(M, C, D, J)$. We first define an affine connection $D^{\prime}$ by

$$
D_{X}^{\prime} Y:=D_{X} Y-\frac{1}{2} J\left(D_{X} J\right) Y \text {. }
$$

Then $D^{\prime}$ preserves $J$ and $C$, i.e., $D^{\prime} J \equiv 0, D^{\prime} g=\omega_{g} \otimes g$. The curvature tensors $R^{\prime}=R^{D^{\prime}}$ and $R^{D}$ satisfy the following relation:

Proposition 2.4.

$$
\begin{aligned}
R^{\prime}(X, Y) V= & \frac{1}{2}\left(R^{D}(X, Y) V-J R^{D}(X, Y) J V\right) \\
& -\frac{1}{4}\left(\left(D_{X} J\right)\left(D_{Y} J\right)-\left(D_{Y} J\right)\left(D_{X} J\right)\right) V .
\end{aligned}
$$

Let $T^{1,0} M$ denote the $\sqrt{-1}$-eigenspace of $J$ in the complexified tangent bundle $T M \otimes C$. Then we can identify $T M$ with $T^{1,0} M$, as complex vector bundle over $M$. The cohomology class of a closed 2-form $\gamma^{\prime}:=\operatorname{Re}\left(\sqrt{-1} \operatorname{tr}_{C}\left(R^{\prime}\right)\right)$ determines the first Chern class $c_{1}(M)$ of $(M, J)$, namely, $2 \pi c_{1}(M)=\left[\gamma^{\prime}\right]$ in $H^{2}(M ; \boldsymbol{R})$, the second cohomology group with real coefficient. By $(2.21)$, we can rewrite $\gamma^{\prime}$ as

$$
\gamma^{\prime}(X, Y)=\rho^{D * \text { (skew) }}(X, Y)-\frac{1}{4} \mathscr{D}(X, Y),
$$

where $\rho^{D *}$ and $\mathscr{D}$ are defined respectively by

$$
\rho^{D *}(X, Y):=r^{D *}(J X, Y), \quad \mathscr{D}(X, Y):=\operatorname{tr}\left(V \mapsto\left(D_{X} J\right)\left(D_{Y} J\right) J V\right) .
$$

From (2.7) in Proposition 2.1, $\mathscr{D}$ is a $J$-invariant 2-form on $M$ satisfying

$$
\mathscr{D} \wedge \Omega_{g}=\frac{1}{4}|D J|_{g}^{2} \Omega_{g}^{2}
$$

By making use of (2.10), we can express $\gamma^{\prime}$ as

$$
\gamma^{\prime}=\rho^{D \text { (skew.nnv) }}+\frac{1}{2} \mathscr{B}-\frac{1}{4} \mathscr{D}+\rho^{D *(\text { skew.anti) }},
$$

where $\mathscr{B}$ is defined by $\mathscr{B}(X, Y):=B(J X, Y)$. Note that $\mathscr{B}$ is a $J$-invariant 2form satisfying

$$
\mathscr{B} \wedge \Omega_{g}=\frac{1}{4}|D J|_{g}^{2} \Omega_{g}^{2} .
$$

In our 4-dimensional case, we can verify the following:

LEMMA 2.5 .

$$
\rho^{D *(\text { skew.nnv })}-\rho^{D(\text { skew.nnv })}\left(=\frac{1}{2} \mathscr{B}\right)=\frac{1}{4}\left(s_{g}^{D *}-s_{g}^{D}\right) \Omega_{g} .
$$


From Proposition 2.2 and the lemma above, we can also rewrite $\gamma^{\prime}$ as

$$
\gamma^{\prime}=\rho_{0}^{D \text { (skew.nv) }}+\frac{1}{4}\left(s_{g}^{D}+\frac{1}{4}|D J|_{g}^{2}\right) \Omega_{g}-\frac{1}{4} \mathscr{D}_{0}+\rho^{D *(\text { skew.anti) }}
$$

where $\rho_{0}^{D \text { (skew.nv) }}$ and $\mathscr{D}_{0}$ denote the components of $\rho^{D \text { (skew.nnv) }}$ and $\mathscr{D}$ orthogonal to $\Omega_{g}$, respectively.

The squared first Chern class $c_{1}^{2}(M)$ is given by $4 \pi^{2} c_{1}^{2}(M)=\left[\gamma^{\prime} \wedge \gamma^{\prime}\right]$ in $H^{4}(M: \boldsymbol{R})$. Identifying $H^{4}(M ; \boldsymbol{R})$ with $\boldsymbol{R}$ via the integration, we obtain the following formula:

PROPOSITION 2.6.

$$
\begin{aligned}
4 \pi^{2} c_{1}^{2}(M)= & \int_{M}\left\{\frac{1}{8}\left(s_{g}^{D}\right)^{2}+\frac{s_{g}^{D}}{16}|D J|_{g}^{2}+\frac{1}{128}|D J|_{g}^{4}\right. \\
& \left.-\left|\rho_{0}^{D \text { (skew.nnv) }}-\frac{1}{4} \mathscr{D}_{0}\right|_{g}^{2}+\left|\rho^{D * \text { (skew.anti) })}\right|_{g}^{2}\right\} \sigma_{g}
\end{aligned}
$$

where $\sigma_{g}$ denotes the volume form of $(M, g)$ (i.e., $\left.\sigma_{g}=(1 / 2) \Omega_{g}^{2}\right)$.

\section{Main result}

In this section, we prove the following result, which is a conformal analogue to the result due to Sekigawa [6]:

THEOREM 3.1. Let $(M, C, D, J)$ be a compact almost Hermitian-EinsteinWeyl 4-manifold. If the conformal scalar curvature $s^{D}$ is nonnegative, then $J$ must be integrable, i.e., $(M, C, D, J)$ is a Hermitian-Einstein-Weyl manifold.

We first recall the following (see Pedersen-Poon-Swann [8]):

Proposition 3.2. Let $(M, C, D)$ be a compact oriented Einstein-Weyl 4manifold. Then the Euler characteristic $\chi(M)$ and the signature $\tau(M)$ satisfy the following:

$$
2 \chi(M)+3 \tau(M)=\frac{1}{4 \pi^{2}} \int_{M}\left\{2\left|W_{+}\right|_{g}^{2}+\frac{1}{24}\left(s_{g}^{D}\right)^{2}+\frac{1}{4}|d \omega|_{g}^{2}\right\} \sigma_{g} .
$$

Notice that for a compact almost complex 4-manifold $M$, the squared first Chern class $c_{1}^{2}(M)$ coincides with the characteristic number $2 \chi(M)+3 \tau(M)$.

Let $(M, C, D, J)$ be an almost Hermitian-Weyl 4-manifold. If it is also Einstein-Weyl, then

$$
\rho_{0}^{D \text { (skew.nnv) }} \equiv 0
$$


Hence the formula (2.23) leads us to another expression of $c_{1}^{2}(M)$ :

$$
\begin{aligned}
4 \pi^{2} c_{1}^{2}(M)=\int_{M}\{ & \frac{1}{8}\left(s_{g}^{D}\right)^{2}+\frac{s_{g}^{D}}{16}|D J|_{g}^{2}+\frac{1}{128}|D J|_{g}^{4} \\
& \left.-\frac{1}{16}\left|\mathscr{D}_{0}\right|_{g}^{2}+\left|\rho^{D * \text { (skew.anti) }}\right|_{g}^{2}\right\} \sigma_{g} .
\end{aligned}
$$

The squared norm of $\mathscr{D}_{0}$ can be calculated as follows. At each point $p$ on $M$, we define a subspace $\mathscr{N}_{p}$ of $T_{p} M$ by $\mathscr{N}_{p}:=\left\{X \in T_{p} M \mid D_{X} J=0\right\}$. It is immediate from Proposition 2.7 that $\mathcal{N}_{p}$ is $J$-invariant and hence has even real dimension. Note that $g\left(\left(D_{X} J\right) Y, V\right)$ is $J$-anti-invariant and skewsymmetric with respect to $Y, V$. Since the real dimension of $\bigwedge^{\text {(anti) }}$ is two, we can write $g\left(\left(D_{X} J\right) Y, V\right)$, at least locally, as

$$
g\left(\left(D_{X} J\right) Y, V\right)=\alpha_{2}(X) \Phi_{2}(Y, V)+\alpha_{3}(X) \Phi_{3}(Y, V),
$$

where $\alpha_{2}, \alpha_{3}$ are local 1-forms and $\left\{\Phi_{2}, \Phi_{3}\right\}$ is a local basis for $\bigwedge^{(\text {anti) }}$. Then $X \in \mathscr{N}_{p}$ if and only if $\alpha_{1}(X)=\alpha_{2}(X)=0$. Counting the dimensions, we see that the real dimension of $\mathcal{N}_{p}$ is not less than two. Take a $g$-orthonormal basis $\left\{e_{1}, e_{2}=J e_{1}, e_{3}, e_{4}=J e_{3}\right\}$ for $T_{p} M$ satisfying $e_{1}, e_{2} \in \mathscr{N}_{p}$. We then obtain $\mathscr{D}\left(e_{i}, e_{A}\right)=0(i=1,2 ; A=1,2,3,4)$. From $J$-invariance of $\mathscr{D}$ and (2.22), the squared norm of $\mathscr{D}_{0}$ is given by $\left|\mathscr{D}_{0}\right|_{g}^{2}=(1 / 8)|D J|_{g}^{4}$. Summarizing these, we obtain the following:

$$
c_{1}^{2}(M)=\frac{1}{4 \pi^{2}} \int_{M}\left\{\frac{1}{8}\left(s_{g}^{D}\right)^{2}+\frac{s_{g}^{D}}{16}|D J|_{g}^{2}+\left|\rho^{D *(\text { skew.anti) }}\right|_{g}^{2}\right\} \sigma_{g} .
$$

We can simplify the term $\left|\rho^{D *(\text { skew.anti) }}\right|_{g}^{2}$ as follows. Let $\left(R^{D}\right)_{g}$ denote the curvature operator on $\bigwedge^{2} T^{*} M$. Namely, it is defined by raising indices of the curvature tensor $R^{D}$ with respect to $g$ :

$$
\left(R^{D}\right)_{g}(\alpha)(X, Y):=\frac{1}{2} \sum_{A, B, I, J} \alpha_{A B} g^{A I} g^{B J} g\left(R^{D}(X, Y) e_{J}, e_{I}\right)
$$

where $\alpha_{A B}$ are the components of a 2-form $\alpha$ with respect to a local frame field $\left\{e_{A}\right\}$ and where $\left(g^{A B}\right)$ denotes the inverse matrix of $g=\left(g_{A B}\right)=\left(g\left(e_{A}, e_{B}\right)\right)$. It should be noted that the 2-form $\left(R^{D}\right)_{g}\left(\Omega_{g}\right)$ is independent of the choice of $g$ in $C$ (i.e., $\left(R^{D}\right)_{g}\left(\Omega_{g}\right)=\left(R^{D}\right)_{g^{\prime}}\left(\Omega_{g^{\prime}}\right)$ for $\left.g, g^{\prime} \in C\right)$. Setting $R^{D}(\Omega):=\left(R^{D}\right)_{g}\left(\Omega_{g}\right)$, we can show the following:

Proposition 3.3. Let $(M, C, D)$ be a Weyl manifold with a compatible almost complex structure $J$. Then we have

$$
R^{D}(\Omega)^{(\text {inv })}=\rho^{D *(\text { skew..nv })}, \quad R^{D}(\Omega)^{(\text {anti) }}=\rho^{D *(\text { skew.anti) }} .
$$


If $(M, C, D, J)$ is an almost Hermitian-Einstein-Weyl 4-manifold, then we obtain

$$
\begin{gathered}
R^{D}(\Omega)^{(\mathrm{inv})}=\frac{1}{4}\left(s_{g}^{D}+\frac{1}{2}|D J|_{g}^{2}\right) \Omega_{g}, \quad R^{D}(\Omega)_{0}^{(\mathrm{inv})} \equiv 0 \\
\rho^{D *(\text { skew.anti) }}=R^{D}(\Omega)-\frac{1}{4}\left(s_{g}^{D}+\frac{1}{2}|D J|_{g}^{2}\right) \Omega_{g} .
\end{gathered}
$$

The formulae (3.5) and (3.6) can be seen from (3.2), Proposition 2.2 and Lemma 2.5 .

Suppose that $(M, C, D, J)$ is an almost Hermitian-Einstein-Weyl 4-manifold. Taking account of (3.6), we have

$$
\left|\rho^{D *(\text { skew.anti) }}\right|_{g}^{2}=\left|R^{D}(\Omega)\right|_{g}^{2}-\frac{1}{8}\left(s_{g}^{D}+\frac{1}{2}|D J|_{g}^{2}\right)^{2} .
$$

If $M$ is compact, we can then rewrite (2.23) as follows:

$$
c_{1}^{2}(M)=\frac{1}{4 \pi^{2}} \int_{M}\left\{-\frac{s_{g}^{D}}{16}|D J|_{g}^{2}-\frac{1}{32}|D J|_{g}^{4}+\left|R^{D}(\Omega)\right|_{g}^{2}\right\} \sigma_{g} .
$$

Comparing (3.7) with (3.1), we therefore obtain the following integral formula:

$$
\begin{aligned}
\int_{M}\{ & \left.-\frac{s_{g}^{D}}{16}|D J|_{g}^{2}-\frac{1}{32}|D J|_{g}^{4}\right\} \sigma_{g} \\
& =\int_{M}\left\{2\left|W_{+}\right|_{g}^{2}+\frac{1}{24}\left(s_{g}^{D}\right)^{2}-\left|R^{D}(\Omega)\right|_{g}^{2}+\frac{1}{4}|d \omega|_{g}^{2}\right\} \sigma_{g} .
\end{aligned}
$$

The following is sufficient to prove our main theorem:

Proposition 3.4. For any compact almost Hermitian-Einstein-Weyl 4manifold $(M, C, D, J)$, the following inequality holds:

$$
\int_{M}\left\{2\left|W_{+}\right|_{g}^{2}+\frac{1}{24}\left(s_{g}^{D}\right)^{2}-\left|R^{D}(\Omega)\right|_{g}^{2}+\frac{1}{4}|d \omega|_{g}^{2}\right\} \sigma_{g} \geq 0 .
$$

If the conformal scalar curvature $s_{g}^{D}$ is nonnegative, then the left hand side of (3.8) is nonpositive; however, from Proposition 3.4, the right hand side of (3.8) is nonnegative. It therefore follows that $|D J|_{g}^{2} \equiv 0$, i.e., $J$ is integrable.

Before proving Proposition 3.4, we first recall that the decomposition (2.2) of $R^{D}$ for an Einstein-Weyl 4-manifold $(M, C, D)$ is given explicitly by

$$
\begin{aligned}
g\left(R^{D}(X, Y) V, U\right)= & g(W(X, Y) V, U)+\frac{s_{g}^{D}}{24} g \bowtie g(X, Y, V, U) \\
& +\frac{1}{4} d \omega \bowtie g(X, Y, V, U)-\frac{1}{2} d \omega \otimes g(X, Y, V, U),
\end{aligned}
$$


where $(ه$ denotes the Kulkarni-Nomizu product:

$$
\begin{aligned}
(t \otimes g)(X, Y, V, U):= & t(X, U) g(Y, V)-t(Y, U) g(X, V) \\
& +t(Y, V) g(X, U)-t(X, V) g(Y, U),
\end{aligned}
$$

for any (0,2)-tensor field $t$. By (3.9), we can show the following:

Lemma 3.5. Let $(M, C, D, J)$ be an almost Hermitian-Einstein-Weyl 4manifold. Then

$$
R^{D}(\Omega)=W(\Omega)+\frac{s_{g}^{D}}{12} \Omega_{g}-\frac{1}{2} J(d \omega)^{(\text {anti) }}
$$

where $J(d \omega)^{(\text {anti) }}(X, Y):=(d \omega)^{(\text {anti) }}(X, J Y)$ and $W(\Omega)$ is defined by replacing $R^{D}$ of $R^{D}(\Omega)$ with $W$.

Proof of Proposition 3.4. Let $\left\{\Phi_{1}, \Phi_{2}, \Phi_{3}\right\}$ be a local orthonormal frame field for $\Lambda_{+}$, the space of self-dual 2 -forms, such that $\Phi_{1}:=\Omega_{g} / \sqrt{2}$ and that $\left\{\Phi_{2}, \Phi_{3}\right\}$ forms an orthonormal basis for $\bigwedge^{(\text {anti) }}$. We may express the self-dual Weyl tensor $W_{+}$as

$$
W_{+}=\left(\begin{array}{lll}
w_{11} & w_{12} & w_{13} \\
w_{12} & w_{22} & w_{23} \\
w_{13} & w_{23} & w_{33}
\end{array}\right) .
$$

By definition, the trace of $W_{+}$vanishes:

$$
\operatorname{tr} W_{+}=w_{11}+w_{22}+w_{33} \equiv 0 .
$$

The squared norm $\left|W_{+}\right|_{g}^{2}$ of $W_{+}$is given by

$$
\left|W_{+}\right|_{g}^{2}=w_{11}^{2}+w_{22}^{2}+w_{33}^{2}+2\left(w_{12}^{2}+w_{13}^{2}+w_{23}^{2}\right) \text {. }
$$

Noting that $W(\Omega)=W_{+}(\Omega)$ and $g\left(J(d \omega)^{(\text {anti) }}, \Omega_{g}\right) \equiv 0$, we can rewrite (3.10) as

$$
\begin{aligned}
R^{D}(\Omega)= & W(\Omega)+\frac{s_{g}^{D}}{12} \Omega_{g}-\frac{1}{2} J(d \omega)^{(\text {anti })} \\
= & \sqrt{2}\left(w_{11}+\frac{s_{g}^{D}}{12}\right) \Phi_{1}+\left(\sqrt{2} w_{12}-\frac{1}{2} g\left(J(d \omega)^{(\text {anti })}, \Phi_{2}\right)\right) \Phi_{2} \\
& +\left(\sqrt{2} w_{13}-\frac{1}{2} g\left(J(d \omega)^{(\text {anti })}, \Phi_{3}\right)\right) \Phi_{3} .
\end{aligned}
$$

From (3.11), we have 


$$
\begin{aligned}
2\left|W_{+}\right|_{g}^{2}+ & \frac{1}{24}\left(s_{g}^{D}\right)^{2}-\left|R^{D}(\Omega)\right|_{g}^{2} \\
= & 2\left(w_{11}^{2}+w_{22}^{2}+w_{33}^{2}\right)+4\left(w_{12}^{2}+w_{13}^{2}+w_{23}^{2}\right)+\frac{1}{24}\left(s_{g}^{D}\right)^{2}-2\left(w_{11}+\frac{1}{12} s_{g}^{D}\right)^{2} \\
& -\left(\sqrt{2} w_{12}-\frac{1}{2} g\left(J(d \omega)^{(\text {anti })}, \Phi_{2}\right)\right)^{2}-\left(\sqrt{2} w_{13}-\frac{1}{2} g\left(J(d \omega)^{(\text {anti })}, \Phi_{3}\right)\right)^{2} \\
= & 2\left\{\left(w_{22}+\frac{1}{12} s_{g}^{D}\right)^{2}+\left(w_{33}+\frac{1}{12} s_{g}^{D}\right)^{2}\right\}+\left(\sqrt{2} w_{12}+\frac{1}{2} g\left(J(d \omega)^{(\text {anti })}, \Phi_{2}\right)\right)^{2} \\
& +\left(\sqrt{2} w_{13}+\frac{1}{2} g\left(J(d \omega)^{(\text {anti })}, \Phi_{3}\right)\right)^{2}+4 w_{23}^{2}-\frac{1}{2}\left|J(d \omega)^{(\text {anti })}\right|_{g}^{2} \\
\geq & -\frac{1}{2}\left|J(d \omega)^{(\text {anti })}\right|_{g}^{2}=-\frac{1}{2}\left|(d \omega)^{(\text {anti })}\right|_{g}^{2} \\
= & -\frac{1}{2}\left|(d \omega)_{+}\right|_{g}^{2} .
\end{aligned}
$$

Here we notice that the last equality can be seen by using (2.4): $d \omega \wedge \Omega_{g} \equiv 0$. Thus we obtain

$$
\begin{aligned}
\int_{M}\left\{2\left|W_{+}\right|^{2}+\frac{1}{24}\left(s_{g}^{D}\right)^{2}-\left|R^{D}(\Omega)\right|_{g}^{2}+\frac{1}{4}|d \omega|_{g}^{2}\right\} \sigma_{g} \\
\geq \int_{M}\left\{-\frac{1}{2}\left|(d \omega)_{+}\right|_{g}^{2}+\frac{1}{4}|d \omega|_{g}^{2}\right\} \sigma_{g} \\
=-\frac{1}{4} \int_{M}\left\{\left|(d \omega)_{+}\right|_{g}^{2}-\left|(d \omega)_{-}\right|_{g}^{2}\right\} \sigma_{g} \\
=-\frac{1}{4} \int_{M} d \omega \wedge d \omega=-\frac{1}{4} \int_{M} d(\omega \wedge d \omega)=0 .
\end{aligned}
$$

This shows the proposition.

\section{Remarks}

It is well-known that for a compact Einstein-Weyl manifold $(M, C, D)$, there exists a metric $g$ in $C$ such that the dual vector field $\omega_{g}^{\#}$ of $\omega_{g}$ is a Killing vector field on $(M, g)$. Such a metric $g$ is unique up to homothety and hence called the standard metric (see Gauduchon [2], Pedersen-Swann [9]). It is also well-known that for a compact Einstein-Weyl manifold $(M, C, D)$, the 1-form $\omega_{g}$ of the standard metric $g$ must vanish if $s^{D}<0$. Thus any compact almost HermitianEinstein-Weyl 4-manifold with negative conformal scalar curvature is determined by an almost Kähler-Einstein structure. 
By virtue of Theorem 3.1, any compact almost Hermitian-Einstein-Weyl 4-manifold with nonnegative conformal scalar curvature must be HermitianEinstein-Weyl (i.e., the almost complex structure is integrable). GauduchonIvanov [3] studied such manifolds and obtained the following:

Proposition 4.1. Let $g$ be the standard metric for a compact HermitianEinstein-Weyl 4-manifold $(M, C, D, J)$. Then the following two cases occur:

(i) $(M, g, J)$ is Kähler-Einstein, or

(ii) $(M, g)$ is locally isometric to $\boldsymbol{R} \times S^{3}$, the Lee form $\beta_{g}$ is $\nabla$-parallel, the Weyl structure $D$ is flat, and $(M, J)$ is a Hopf surface, where $\nabla$ denotes the Levi-Civita connection of $(M, g)$.

From Theorem 3.1 and Proposition 4.1, we obtain

Corollary 4.2. A compact almost Hermitian-Einstein-Weyl 4-manifold, which is not determined by any almost Kähler-Einstein structure, must be a Hermitian-Einstein-Weyl manifold of type (ii) in Proposition 4.1.

We finally remark on higher dimensional cases. Let $(M, C)$ be a compact conformal manifold of real dimension $2 n(>4)$ with a compatible almost complex structure $J$, and $D$ the canonical Weyl connection of $(M, C, J)$. Suppose that the condition (2.3) is satisfied (i.e., $d \Omega_{g}=\beta_{g} \wedge \Omega_{g}$ ). Then the Lee form $\beta_{g}$ is automatically closed, and hence $(M, C, D, J)$ is determined by a locally conformal almost Kähler (1.c.a.K.) structure, and vice versa (see Vaisman [11]).

If $(M, C, D)$ is also Einstein-Weyl, then $(M, C, D, J)$ is determined by a locally conformal almost Kähler-Einstein structure. Let $g$ be the standard metric for $(M, C, D, J)$. By the closedness of the Lee form $\beta_{g}$, the dual vector field $\beta_{g}^{\#}$ of $\beta_{q}$ is parallel with respect to the Levi-Civita connection $\nabla$ of $(M, g)$. Then the conformal scalar curvature $s_{g}^{D}$ is constant, since $s_{g}^{D}$ is a harmonic function on $(M, g)$. In particular, the sign of $s^{D}$ is well-defined (see Pedersen-Swann [10]). We further suppose that $s^{D}$ is nonnegative. If $\beta_{g} \equiv 0$, then $(M, g, J)$ is an almost Kähler-Einstein manifold with nonnegative scalar curvature. From Sekigawa's result [7], $(M, g, J)$ is in fact Kähler-Einstein.

In the case where $s^{D}>0$, the Ricci curvature of $(M, g)$ is strictly positive. From Myers' theorem, the fundamental group $\pi_{1}(M)$ is finite, and hence the first Betti number $b_{1}(M)$ vanishes. We therefore obtain $\beta_{g} \equiv 0$, since $\beta_{g}$ is a harmonic 1-form on $(M, g)$. In the case where $s^{D} \equiv 0$, we may assume that $\beta_{g} \neq 0$. Then the standard argument tells us $b_{1}(M)=1$ (see Pedersen-Swann $[10])$.

On the other hand, Kashiwada [5] studied the integrability problem for an almost generalized Hopf manifold, which means a locally conformal almost Kähler manifold $(M, g, J)$ with parallel Lee form $\beta_{g}$ satisfying that $J \beta_{g}^{\#}$ is a Killing vector field on $(M, g)$. If $J$ is also integrable, then $(M, g, J)$ is called a generalized Hopf manifold. Notice that for a locally conformal Kähler manifold $(M, g, J)$, the vector field $J \beta_{g}^{\#}$ is automatically a Killing vector field on $(M, g)$ if 
$\beta_{g}$ is parallel. For convenience, we regard (almost) Kähler-Einstein manifolds as (almost) generalized Hopf manifolds with vanishing Lee forms. $[5]:$

The following is an immediate consequence from a result due to Kashiwada

Proposition 4.3. Let $(M, g, J)$ be a compact almost generalized Hopf manifold of dimension grater than four. Suppose that its canonical Weyl structure $(C, D)$ is Einstein-Weyl. If the conformal scalar curvature $s^{D}$ is nonnegative, then $J$ must be integrable, i.e., $(M, g, J)$ is a generalized Hopf manifold.

Acknowledgment. The author would like to thank Professor Toyoko Kashiwada for calling his attention to this direction and for comments. The author would also like to thank Professor Kouei Sekigawa for sending him Draghici's paper, and thank the referee for suggestions.

\title{
REFERENCES
}

[1] T. DraghicI, Special metrics on symplectic manifolds, PhD Thesis, Michigan State Unıv. 1997.

[2] P Gauduchon, La 1-forme de torsion d'une variété hermitienne compacte, Math. Ann., 267 (1984), 495-518.

[3] $\mathbf{P}$ Gauduchon and S. Ivanov, Einsteın-Hermitıan surfaces and Hermitıan Einstein-Weyl structures in dimension 4, Math. Z., 226 (1997), 317-326.

[4] M. Iтон, Compact Einstein-Weyl manifolds and the associated constant, Osaka J. Math., 35 (1998), 567-578.

[5] T. Kashiwada, On $\alpha \beta$-Einstein almost generalized Hopf manifolds, Nat. Sc1. Rep. Ochanomizu Unıv., 46 (1995), 1-7

[6] K. Sekigawa, On some 4-dimensional compact Einstein almost Kähler manifolds, Math. Ann., 271 (1985), 333-337

[ 7 ] K. Sekigawa, On some compact Einsteın almost Kähler manifolds, J. Math. Soc. Japan, 39 (1987), 677-684.

[ 8 ] H. Pedersen, Y S. Poon and A. Swann, The Hitchın-Thorpe inequality for Einstein-Weyl manifolds, Bull. London Math. Soc., 26 (1994), 191-194.

[9] H. Pedersen and A. Swann, Riemannian submersions, four-manifolds and Einstein-Weyl geometry, Proc. London Math. Soc. (3), 66 (1993), 381-399.

[10] H. Pedersen and A. Swann, Einstein-Weyl geometry, the Bach tensor and conformal scalar curvature, J. Reine Angew. Math., 441 (1993), 99-113.

[11] I. VAISMAN, On locally conformal almost Kähler manifolds, Israel J. Math., 24 (1976), 338 351.

\author{
Numazu College of Technology \\ 3600 OOKA, NumazU-SHI \\ SHIZUOKA 410-8501 \\ JAPAN \\ E-mail: kamada@1a.numazu-ct.ac.jp
}

